\title{
LIMIT EQUILBRIUM SAFETY ANALYSIS OF PILE IN LATERAL SPREAD: THREE-LAYER
}

\author{
Department of Structures and Metallic Constructions, Housing \& Building \\ Research Center, 87 El Tahrir St. Dokki, Giza, Egypt. \\ Reda Farag ${ }^{1}$ \\ E-mail: red_bordany@yahoo.com
}

\begin{abstract}
The large amount of uncertainties in liquefaction-induced lateral spreading particularly leads to pile damage after many earthquakes. This paper presents a simplified safety analysis method of the single piles subjected to lateral spread in three layered soils. With the suggested method the analyst can get a quick estimate of the pile safety using few simulations of the pile-soil structural model. The method integrates a limit equilibrium-based finite element model with the response surface method as well as the first or the second order reliability method (FORM/SORM). In the finite element model, the assumptions of limit equilibrium method are simply applied. The soil is represented by 3D solid elastoplastic (Drucker-Prager failure criterion) while the pile is represented by an elastic 3D beam element. The simplified procedure not only estimates the safety but also determines the most sensitive basic pile and soil parameters affecting the response. Both serviceability and ultimate limit states are applicable. The accuracy of the proposed analytical method is evaluated against the Monte Carlo Simulation Method. At the beginning, two simple examples are used to verify, validate and further illustrate the method. After that, a more complex example is used to substantiate the superiority of the method. The effect of numerous uncertainties associated with the system is taken into account.
\end{abstract}

Keywords: Lateral spread, three layered soil system, limit equilibrium, safety

\section{Introduction}

Pile sited on poorly consolidated natural deposits or fills that are particularly susceptible to liquefaction and lateral spread, are particularly vulnerable to ground displacement. The safety against the potential lateral ground displacement is surrounded by a lot of uncertainties in the design and environment variables. Moreover, there is a considerable difficulty in obtaining undisturbed samples of loose granular (liquefiable) sediment for laboratory evaluation of constitutive soil properties. As a result, the uses of analytical methods, which rely on accurate measurements of constitutive properties, are usually limited to critical projects or to research. This complexity increases the value of the information those can be provided by the advanced analysis methods. Moreover, it had encouraged many simplified methods of analysis to be sought such as Beam-on-Winkler-Springs, Bradley, et al (2011), pseudo-static method, Tabesh and Poulos (2001) and the limit equilibrium method, Dobry, et al (2003).

Bradley, et al (2011), have proposed a probabilistic framework for Pseudostatic analysis of pile foundations in liquefied and lateral spreading soils. A pseudo-static method involves applying static displacements and forces to a typical beam-spring model, has been used in Monte Carlo simulation. It has been observed that the significant uncertainties in the Pseudostatic analysis result in significant uncertainty in both pile-head displacement and pile bending moment for a given level of input ground motion. Consequently it has been reported that decision making based on a single reference model is potentially erroneous.

In the present paper, the safety of pile under lateral spread in three layer soil profile is estimated using a proposed method. (Pile in two layer soil profile is manipulated in an ongoing paper).The method consists of a limit equilibrium-based finite element model coupled with the response surface method (RSM) as well as the first or second order reliability method (FORM/SORM). The limit state 
functions are formulated in terms of the random variables that affect the structural design of pile under lateral spread. Using serviceability and ultimate limit state functions, first or second order reliability methods, FORM/SORM have been used for safety/reliability calculations. The results are verified using Monte Carlo Simulation Method. Furthermore, the most important random variables are determined.

\section{Limit Equilibrium Approach}

As the main objective of the present paper is to introduce an approach to compute a quick estimation of the safety information using not so many simulations of the structural model, a simple limit equilibrium approach (LE) that was introduced by Dobry, et al (2003), is used. The basic principle of this method is that the lateral forces applied by the soil against a given deep foundation are limited by the maximum pressure that can be applied by the liquefied soil (case of two-layer soil profile) or by the passive resistance of the non-liquefied layers (case of three-layer-soil profile). This method provides upper bound for the bending moments, displacements and rotations of the pile foundation, and is especially useful as an engineering tool for design and retrofitting decision. As reported by Dobry, et al (2003), the limit equilibrium, LE analysis has been used to explain the excellent performance of a bridge foundation to lateral spreading induced by the 1987 Edgecumbe earthquake in New Zealand; and by Japan Road Association Association (1996) and Yokoyama, et al (1997), to evaluate the response of bridge foundations to lateral soil deformation during the Kobe earthquake.

Relying typically on laboratory test result, Dobry, et al (2003) had implemented a calibration of the LE method for a wide range of single pile conditions in two- and three-layer soil profiles using centrifuge tests. A limit equilibrium method is proposed to evaluate the bending response of floating and end-bearing pile foundation subjected to actual lateral spreads in the field. Two backfigured values; soil pressure $(p=10.3 \pm 1.5(15 \%) \mathrm{kPa})$ and the rotational flexibility $\left(k_{r}=5738 \mathrm{kN} \mathrm{m} / \mathrm{rad}\right)$ were used to drive analytical solutions for elastic beams (pile). Figure 1, shows a typically pile under lateral spread in two and three layer soil profile, while Figure 2-a shows the LE model of threelayered soils.

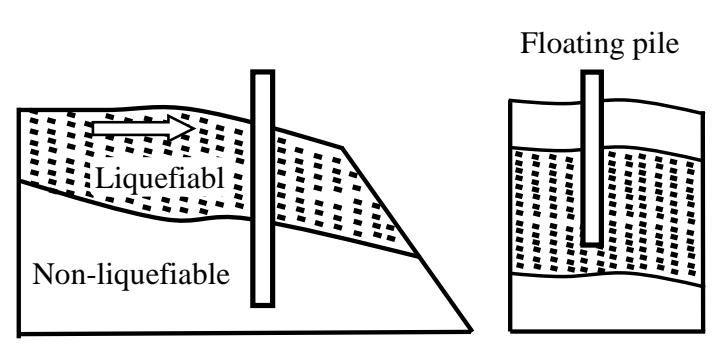

a) Pile in 2-layer soil profile

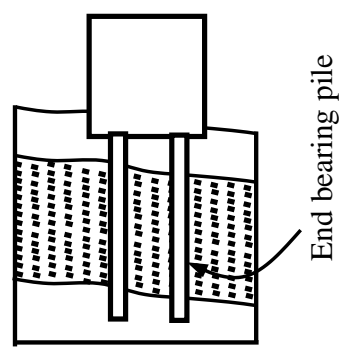

b) Pile in 3-layer soil profile

\section{Figure 1. Pile foundation in laterally spreading ground Pile}

\subsection{Pile in Three-layer Soil Profile}

In this system, the liquefiable soil layer is sandwiched between two non-liquefiable layers. As the pile in three-layer is more complicated, the LE assumes that, the displaced pile shape has double curvature and the maximum positive and negative moment occur simultaneously at the top and bottom of the liquefied layer. They occur due to the largest strength of the upper and lower layer. Moreover, the effect of the liquefied layer is negligible compared with the effect of the non-liquefied layers. The loading history involves two stages -in the upper non liquefied layer-; elastic and elastic perfectly plastic. The present work manipulates only the elastic stage. 


\subsection{Elastic stage}

It is assumed in this stage -of low or small lateral displacement $(D H \cong 0.0-0.3 \mathrm{~m}$ )- that the soil in both top and bottom non-liquefiable layers can be represented by rotational spring, $k_{r}=5738 \mathrm{kN}$ $\mathrm{m} / \mathrm{rad}$, as shown in Figure 2-a. Moreover, the values of moment at top and bottom boundaries of the liquefied layer are equal, $M_{t}=M_{b}$

$$
M_{t}=\frac{D_{H}}{L_{L i q}\left(L_{L i q} / 6 E I+1 / k_{r}\right)}
$$

Where $L_{\text {liq }}$, the thickness of the liquefiable soil layer; $E, I$ are the pile elastic modulus and moment of inertia, respectively. The other variables are defined before.

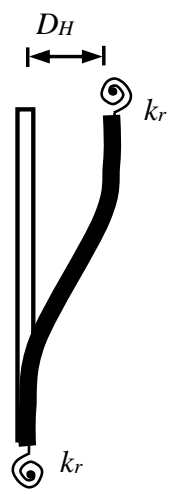

a) Partially fixed ended beam

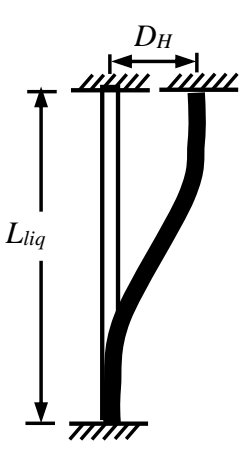

b) Fixed ended beam
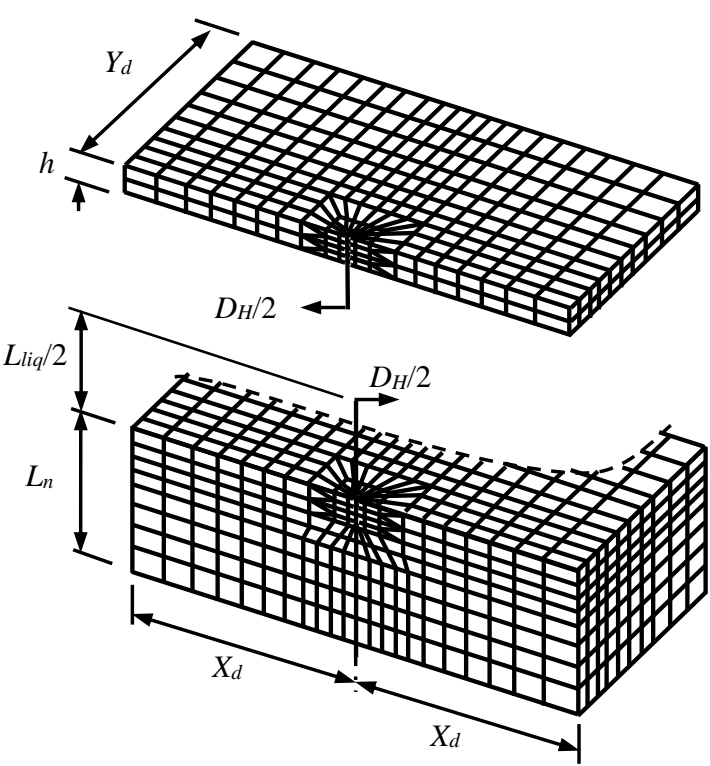

c) LE-based finite element model

Figure 2. Models of 3-Layer

\subsubsection{Simplified elastic beam relation}

If the top and bottom layers are assumed to be rock and the pile is completely fixed in these two layers, as shown in Figure 2-b, then, the above equation is reduced to

$M_{t}=M_{b}=6 E I D_{H} / L_{L i q}^{2}$

\section{4 . Proposed LE-Based FE Model}

As mentioned above, two backfigured variables; pressure and the rotational flexibility are used to derive analytical solutions for elastic beams. In the present paper, a limit equilibrium -based finite element model (LEFE) is proposed. In this model, the non-liquefied layer is represented by a three dimensional nonlinear elasto-plastic (Drucker-Prager) elements, while the pile is represented by elastic 3D beam elements. As, the system is assumed to be symmetrical about the mid thickness of the liquefied layer, one half is used in the analysis. Instead of displacement of the top non-liquefied soil layer by displacement $D_{H}, D_{H} / 2$ is applied to the beam element of each half, as shown in Figure 
2-c. It should be mentioned that, the Drucker-Prager element is represented by the angle of internal friction $(\phi)$; soil cohesion $(c)$; elastic modulus $\left(E_{s}\right)$ and the soil density $\left(\gamma_{s}\right)$. The standalone FE code, COSMOS/M Structural (2000) is used.

\section{5 . Response Surface Method}

Coupling the MCS Method with pile under lateral spread is often prohibited by too long simulations. The response surface method (RSM) is commonly used to approximately generate expressions for the performance functions. Then, FORM/SORM integrated with the RSM to evaluate the safety. The RSM can be found in the literature, Haldar and Mahadevan (2000a). First order polynomial is often used to perform preliminary analysis, Eq. (3). While Second-order polynomial is generally used to represent a response surface, Eq. (4). Mathematically, it can be expressed as:

$$
\begin{aligned}
& \hat{g}(\mathbf{X})=b_{0}+\sum_{i=1}^{n} b_{i} X \\
& \hat{g}(\mathbf{X})=b_{0}+\sum_{i=1}^{n} b_{i} X+\sum_{i=1}^{n} b_{i i} X^{2}
\end{aligned}
$$

where $\mathrm{X}_{\mathrm{i}}(i=1,2, \ldots, k)$ is the $i^{\text {th }}$ random variable, and $b_{0}, b_{i}, b_{i i}$, and $b_{i j}$ are unknown coefficients to be determined from the deterministic analyses of the problem at specific data points, commonly known as experimental sampling points. The number of unknown parameters are $\mathrm{pp}=(k+1)(k+2) / 2$, for Eq. (3). The sampling points are generally selected using some multiple of the standard deviation of the random variables according as:

$$
X_{i}=X_{i}^{C} \pm h_{i} \sigma_{x_{i}} \quad i=1,2, \ldots, k
$$

where $X_{i}$ is the coded $i^{\text {th }}$ variable, $X_{i}^{C}$ and $\sigma_{x_{i}}$ are the coordinates of the centre point and the standard deviation of a random variable $\mathrm{Xi}$, respectively; $h_{i}$ is an arbitrary factor that defines the experimental region, and $k$ is number of random variables in the formulation.

Selection of the center point around which the sampling points are selected is the next task in RSM. The initial center point $x_{c_{1}}$ is selected to be the mean values of the random variable Xi's. Then, using the values of $\mathrm{g}(\mathrm{X})$ obtained from the deterministic FEM evaluations for all the experimental sampling points around the center point, the response surface $\hat{g}_{1}(X)$ can be generated explicitly in terms of the random variables $X$. Once a closed form of the limit state function, $\hat{g}_{1}(X)$, is obtained, the coordinates of the checking point $x_{D_{1}}$ can be estimated using FORM/SORM. The actual response can be evaluated again at the checking point $x_{D_{1}}$, i.e., $\mathrm{g}\left(x_{D_{1}}\right)$ and a new center point $x_{c_{2}}$ can be selected using linear interpolation from the center point $x_{c_{1}}$ to $x_{D_{1}}$ such that $\mathrm{g}(\mathrm{X})=0$. This iterative strategy can be repeated until convergence is met.

\section{6 . Soil-Pile Statistical Properties}

In the present work, piles of reinforced concrete or polyetherimide ULTEM 1000, -used in centrifuge tests- are used in the analysis. The pile modulus of elasticity, $(E)$, the cross sectional area of the pile expressed in the external radius $(r)$ and thickness $(t)$ are considered to be random variables. Besides the pile unit density $(\gamma)$ and Poisson's ratio $(v)$ as in (JCSS)Joint (2000). The modelling of 
uncertainties in geotechnical soil properties are widely reported in the literature. In the present study, the soil elastic modulus $\left(E_{s}\right)$, the cohesion strength $(c)$, the angle of internal friction $(\phi)$, the soil unit density $\left(\gamma_{s}\right)$ and the soil Poisson's ratio $\left(v_{s}\right)$ are considered to be random variables (JCSS)Joint (2006). The uncertainty in the lateral displacement depends on the uncertainties in both soil properties and the earthquake characteristics including accelerations, time histories, duration, etc. In the present work, the lateral displacement is assumed to follow the probability distribution of extreme value Type 1 (EV-I). The statistical characteristics are gathered from the literature for each example.

\section{Numerical Examples}

The proposed method is demonstrated through the following simple examples. The examples are; Simplified Elastic Beam Model; Centrifuge Test Model and Limit Equilibrium Finite Element model. The superiority of the suggested methods is illustrated in example 3. The MCS Method is used for the sake of verification as it is seen next.

\subsection{Example 1: Simplified Elastic Beam Model}

An assumed reinforced concrete pile driven in a liquefiable layer sandwiched between two nonliquefiable layers is considered. The thickness of the liquefiable soil layer $L_{l i q}=7.00 \mathrm{~m}$, and the top layer is subjected to lateral displacement $D_{H}=5 \mathrm{~cm}$. The statistical description of the uncertainties associated with the random variables is listed in Table 1.

Assuming $f$ is the pile flexural strength, the flexural limit state is:

$$
g(\mathbf{X})=f-M_{t} \times r / I=f_{c}-6 E I D_{H} / L_{L i q}^{2} \times r / I
$$

Table 1: Statistical characteristic of random variables - Example 4

\begin{tabular}{|l|l|c|c|c|c|l|l|}
\hline & $\begin{array}{l}\text { Random } \\
\text { variables }\end{array}$ & $\begin{array}{l}\text { Symb } \\
\text { ol }\end{array}$ & $\begin{array}{c}\text { Distributi } \\
\text { on }\end{array}$ & Nominal & Mean & COV & Ref. \\
\hline 1 & $\begin{array}{l}\text { Lateral } \\
\text { spread }\end{array}$ & $D_{H}$ & EV-I & $0.03 \mathrm{~m}$ & $0.03 \mathrm{~m}$ & 0.20 & $*$ \\
\hline 2 & Radius & $r$ & $\mathrm{LN}$ & $0.15 \mathrm{~m}$ & 0.15 & 0.10 & Bednar (1986) \\
\hline 3 & Length & $L_{\text {Liq }}$ & $\mathrm{N}$ & $7.00 \mathrm{~m}$ & 7.00 & 0.04 & ${ }^{*}$ \\
\hline 4 & $\begin{array}{l}\text { E-modulus of } \\
\text { R.C. }\end{array}$ & $E$ & $\mathrm{LN}$ & $\begin{array}{l}2.0 \times 10^{7} \\
\mathrm{kN} / \mathrm{m}^{2}\end{array}$ & $2.01 \times 10^{7}$ & 0.18 & ${ }^{*}$ \\
\hline 5 & R.C. strength & $f_{c}$ & $\mathrm{LN}$ & $\begin{array}{l}22500 \\
\mathrm{kN} / \mathrm{m}^{2}\end{array}$ & 22500 & 0.15 & $\begin{array}{l}\text { Ellingwood } \\
(1980)\end{array}$ \\
\hline
\end{tabular}

* Data not available. Assumed parameters are based on engineering judgment.

Table 2: Results of reliability analysis- Example 4

\begin{tabular}{|l|l|c|c|c|c|c|c|c|c|}
\hline & & \multicolumn{5}{|c|}{ Variables sensitivities } & \multirow{2}{*}{$\beta$} & $P_{f}$ & $\begin{array}{c}\text { function calls } \\
\text { no. }\end{array}$ \\
\hline 1 & & $f_{c}$ & $D_{H}$ & $r$ & $E$ & $L_{\text {Liq }}$ & & & \\
\hline 2 & Sonte Carlo & & & & & & 2.212 & $1.35 \times 10^{-2}$ & $10^{5}$ \\
\hline 3 & $\begin{array}{l}\text { SORM } \\
\text { surface Response } \\
\text { Quadratic } \\
\text { polynomial }\end{array}$ & 0.421 & - & - & - & 0.231 & 2.209 & $1.36 \times 10^{-2}$ & 1 \\
\hline
\end{tabular}


The safety index using Monte Carlo simulation and SORM, $\beta$-MCS and $\beta$-SORM equal 2.212 and 2.209, as listed in Table 2. Using the response surface method, the safety index, $\beta$-index $=1.845$, this value is $16.5 \%$ less than that of Monte Carlo simulation method. The sensitive random variables are; lateral displacement, pile radius and the pile E-modulus with relative importance; $39.6 \%, 53.5 \%$ and $40.1 \%$ respectively, as listed in Table 2.

\subsection{Example 2: Simplified Elastic Beam Model}

A $10 \mathrm{~m}$ soil deposit and pile length is studied in this example. The deposit has $6 \mathrm{~m}$ layer liquefiable sand encased between $2 \mathrm{~m}$ top and $2 \mathrm{~m}$ bottom of non-liquefiable soil. The top layer is subjected to lateral displacement $D_{H}=0.15 \mathrm{~m}$. The pile has a circular section of radius $30 \mathrm{~cm}$ and a bending stiffness $E I=8000 \mathrm{kNm} 2$. This example is the actual model of a centrifuge test (model 1) in Dobry, et al (2003). The pile is manufactured of polyetherimide ULTEM 1000. Assuming that the modulus of elasticity and the flexural strength $E=3300$ and $f=160 \mathrm{Mpa}$, respectively, the pile thickness is found to be $t=3.4 \mathrm{~cm}$, (ULTEM $®$ PEI Resin Product Guide Eng/6/2003 CA).

As this model often simulate a reinforced concrete pile, the value of $f_{c}=22500 \mathrm{kN} / \mathrm{m} 2$ is used in the limit state formulation and in the subsequent analysis. The values of the random variables as well as their statistical properties are gathered from the literature for both the pile and soil and listed in Table 3.

i) Response surface solution:

The analysis is started performing reliability analysis using the linear polynomial, it is observed

Table 3: Statistical Characteristic of Random Variables - Example 5

\begin{tabular}{|l|l|c|c|c|c|c|c|l|}
\hline & Random variables & Sym. & Dist. & Nominal & Mean & Bias & COV & Ref. \\
\hline 1 & Lateral displacement & $D_{H}$ & EV-I & $0.15 \mathrm{~m}$ & 0.15 & 1.0 & 0.20 & ${ }^{*}$ \\
\hline 2 & Radius & $r$ & $\mathrm{LN}$ & $0.30 \mathrm{~m}$ & 0.30 & 1.0 & 0.10 & Bednar (1986) \\
\hline 3 & Thickness & $t$ & $\mathrm{LN}$ & $3.4 \mathrm{~cm}$ & 3.4 & 1.0 & 0.05 & Bednar (1986) \\
\hline 4 & Length & $H_{\text {Liq }}$ & $\mathrm{N}$ & $6.00 \mathrm{~m}$ & 6.00 & 1.0 & 0.04 & ${ }^{*}$ \\
\hline 5 & $\begin{array}{l}\text { Elastic modulus of } \\
\text { pile }\end{array}$ & $E$ & $\mathrm{LN}$ & $3300 \mathrm{Mpa}$ & 3300 & 1.0 & $0.06^{*}$ & $*^{*}$ \\
\hline 6 & Rotational spring & $k_{r}$ & $\mathrm{LN}$ & $5738 \mathrm{kN} \mathrm{m} / \mathrm{rad}$ & 5738 & 1.0 & $0.21^{*}$ & $\begin{array}{l}\text { Dobry, et al } \\
(2003)\end{array}$ \\
\hline 7 & Flexural strength & $f_{c}$ & $\mathrm{LN}$ & $22500 \mathrm{kN} / \mathrm{m}^{2}$ & 22500 & 1.0 & $0.15^{*}$ & Joint (2000) \\
\hline
\end{tabular}

Data not available. Assumed parameters are based on engineering judgment. 
Table 4: Results of Reliability Analysis - Example 5

\begin{tabular}{|c|c|c|c|c|c|c|c|c|c|c|}
\hline & \multicolumn{7}{|c|}{ Variables sensitivities } & \multirow[t]{2}{*}{$\beta$} & \multirow[t]{2}{*}{$P_{f}$} & \multirow{2}{*}{$\begin{array}{c}\text { no. of } \\
\text { calls }\end{array}$} \\
\hline & $f_{c}$ & $\overline{D_{H}}$ & $r$ & $k_{r}$ & $L_{L i q}$ & $t$ & $E$ & & & \\
\hline $\begin{array}{l}\text { i) Response surface } \\
\text { First order polynomial } \\
\text { Second order }\end{array}$ & $\begin{array}{l}0.424 \\
0.433\end{array}$ & $\begin{array}{l}-0.729 \\
-0.755\end{array}$ & $\begin{array}{l}0.433 \\
0.396\end{array}$ & $\begin{array}{l}-0.282 \\
-0.251\end{array}$ & $\begin{array}{l}0.115 \\
0.119\end{array}$ & $\begin{array}{l}0.080 \\
0.082\end{array}$ & $\left|\begin{array}{l}-0.051 \\
-0.051\end{array}\right|$ & $\begin{array}{l}2.521 \\
2.601\end{array}$ & $\begin{array}{l}5.84 \times 10^{-3} \\
4.64 \times 10^{-3}\end{array}$ & $\begin{array}{l}13 \\
13\end{array}$ \\
\hline Scheme 0 & 0.501 & -0.747 & 0.219 & -0.295 & 0.194 & & & 2.666 & $3.83 \times 10^{-3}$ & 9 \\
\hline $\begin{array}{l}\text { ii) Explicit limit state } \\
\text { MCS-6vars }\end{array}$ & & & & & & & & 2.685 & $3.63 \times 10^{-3}$ & $10^{5}$ \\
\hline SORM-6vars & -0.459 & -0.773 & 0.199 & -0.324 & 0.187 & -0.064 & 0.091 & 2.689 & $3.56 \times 10^{-3}$ & 1 \\
\hline
\end{tabular}

that, $E$ and $t$ can be considered as deterministic (their sensitivities are small $(<8 \%)$, reducing the number of variables to four variables as shown in row number 1 of Table 4 . Then applying the quadratic polynomial, the safety index, $\beta$-index $=2.666$. The most important variables are found to be; lateral displacement $\left(D_{H}\right)$, pile radius $(r)$, the rotational flexibility $\left(k_{r}\right)$ and the thickness of the liquefied layer with relative importance; $74.7 \% ; 21.9 \% ; 29.5 \%$ and $19.4 \%$, respectively.

\section{ii) Explicit Limit state}

Using the explicit limit state, it is found that the Monte Carlo simulation safety index and the second order safety index $\beta$-MCS $=2.685$ and $\beta$-SORM $=2.689$, respectively. The safety index of response surface is $0.7 \%$ less than that value of Monte Carlo Simulation. The sensitivities of the variables can be also compared. For example, the sensitivity of the lateral displacement is; 0.755 and 0.773 for the response surface methodology and the explicit limit state, respectively. It can be noted that the most important variables are the same in both cases.

\subsection{Example 3: Simplified Elastic Beam Model}

The pile in the above example (example 2), is subjected to $20 \mathrm{~cm}$ lateral displacement. The suggested LE-based FE model, (LEFE), is used in the analysis. Table 5 shows the statistical properties of the concerned variables. The stochastic model involves 11 variables and the concrete flexural strength is assumed to be $3750 \mathrm{kN} / \mathrm{m} 2$.

The analysis is started by setting up the FE model. In the model $D_{H} / 2$ applies to each half. $M_{b}=40.4$ kN.m. This value is $6.2 \%$ less than the value of LE approach, Dobry, et al (2003). Therefore, the uncertainty in the model bending moment is taken into account through a model factor $\alpha_{b}$.

Following the same procedure as in the previous example, a preliminary analysis is performed using first order polynomial. The large number of variables is reduced to 3 variables; pile radius, $(r)$, the 
lateral displacement, $\left(D_{H}\right)$, and the pile thickness, $(t)$ with sensitivities; $0.065 ; 0.827$ and 0.082 ; respectively. Then, the reliability analysis is preformed using the quadratic polynomial.

\begin{tabular}{|c|l|l|r|r|c|c|c|l|l|}
\hline & & Random variables & Sym. & Dist. & Nominal & Mean & Bias & COV & Ref. \\
\hline 1 & Load & $\begin{array}{l}\text { Lateral } \\
\text { displacement }\end{array}$ & $D_{H}$ & EV-I & $0.2 \mathrm{~m}$ & 0.2 & 1.0 & $0.25^{*}$ & \\
\hline 2 & Pile & $\begin{array}{l}\text { Pile } \\
\text { modulus }\end{array}$ & $E$ & LN & $3300 \mathrm{Mpa}$ & 3300 & 1.0 & $0.06^{*}$ & \\
\hline 3 & & Poisson's ratio & $v$ & $\mathrm{LN}$ & 0.2 & 0.2 & 1.0 & 0.10 & $*$ \\
\hline 4 & & Density & $\gamma$ & $\mathrm{N}$ & $16 \mathrm{kN} / \mathrm{m}^{3}$ & 16 & 1.0 & 0.10 & $*$ \\
\hline 5 & & Radius & $r$ & $\mathrm{LN}$ & $0.30 \mathrm{~m}$ & 0.30 & 1.0 & 0.10 & Bednar (1986) \\
\hline 6 & & Thickness & $t$ & $\mathrm{LN}$ & $3.4 \mathrm{~cm}$ & 3.4 & 1.0 & 0.05 & Bednar (1986) \\
\hline 7 & & Length & $L_{\text {Liq }}$ & $\mathrm{N}$ & $6.00 \mathrm{~m}$ & 6.00 & 1.0 & $0.04^{*}$ & \\
\hline 8 & Top layer & Soil E-modulus & $E_{s}$ & $\mathrm{LN}$ & $\begin{array}{c}1500 \\
\mathrm{kN} / \mathrm{m}^{2}\end{array}$ & 1725 & 1.15 & $0.21^{*}$ & Joint (2006) \\
\hline 9 & & Friction angle & $\phi$ & $\mathrm{LN}$ & $34.5^{\circ}$ & 35.535 & 1.03 & 0.20 & Joint (2006) \\
\hline 10 & & Cohesion strength & $c$ & $\mathrm{LN}$ & $5.1 \mathrm{kN} / \mathrm{m}^{2}$ & 5.61 & 1.10 & 0.37 & Joint (2006) \\
\hline 11 & & Poisson's ratio & $v_{s}$ & $\mathrm{LN}$ & 0.30 & 0.30 & 1.0 & 0.10 & Joint (2006) \\
\hline 12 & & Soil density & $\gamma_{s}$ & $\mathrm{LN}$ & $17 \mathrm{kN} / \mathrm{m}^{2}$ & 17 & 1.0 & 0.10 & Joint (2006) \\
\hline 13 & & Flexural strength & $f_{c}$ & $\mathrm{LN}$ & 3750 & 3750 & 1.0 & 0.15 & Joint (2006) \\
\hline 14 & Model & Bending moment & $\alpha_{b}$ & $\mathrm{~N}$ & 1.00 & 1.00 & 1.00 & 0.10 & $*$ \\
\hline
\end{tabular}

The safety index is found to be $\beta$-index $=1.618$. The relative importance of the above mentioned three important variables are; $48.5 \%, 29.6 \%$ and $9.5 \%$, respectively. These safety results are listed in Table 6.

In this example, more variables related to pile and soil can be taken into account. The number of the considered variables is 14 variables as listed in Table 5.

The proposed method lasts little time. However, it is approximated as it is based on the limit equilibrium method. So, it is appropriate for the preliminary analysis or the not critical projects.

Table 5: Statistical characteristic of random variables - Example 6

*Data not available. Assumed parameters are based on engineering judgment 
Table 6: Results of reliability analysis- Example 6

\begin{tabular}{|l|l|c|c|c|c|c|c|c|l|}
\hline & & \multicolumn{5}{|c|}{ Variables sensitivities } & \multirow{2}{*}{$\beta$} & $P_{f}$ & function \\
& & $f$ & $\alpha_{b}$ & $r$ & $D_{H}$ & $t$ & & & \\
& & & & & & & & & \\
\cline { 1 - 8 } 1 & & & & & & & & & \\
& First order & 0.459 & -0.284 & -0.065 & -0.827 & 0.082 & 2.970 & $9.61 \times 10^{-2}$ & 25 \\
& Second order & 0.424 & -0.267 & -0.820 & -0.262 & 0.093 & 2.404 & $8.11 \times 10^{-3}$ & 7 \\
\hline 2 & Scheme 0 & 0.692 & -0.434 & -0.485 & -0296 & 0.095 & 1.618 & $5.28 \times 10^{-2}$ & 7 \\
\hline
\end{tabular}

\section{Conclusion}

The present paper introduces a simplified method to get a quick estimate value of the safety of pile under lateral spread. In the suggested method a limit equilibrium method based on static equilibrium and kinematic consideration has been coupled with the classical response surface method and the second order reliability method. The method is applied to single pile in three-layer soil profiles. Furthermore, the variables sensitivities are identified. This approximated method extracts quick estimate of reliability information using tens of limit equilibrium-based finite element model. For the three considered examples, it is found that the most important variables are; the pile radius and the lateral displacement.

\section{Nomenclature}

$b_{0}, b_{i}, b_{i i}$, and Unknown coefficients of a polynomial to be determined.

$b_{i j}$

$C$

$D_{H}$

$D_{p}$

$E, E_{s}$

EI

$f, f_{c}$

$g_{f}(x), g_{u x}(x)$

$\hat{g}(\mathbf{X})$

$\hat{g}_{b}(\mathbf{X}), \hat{g}_{u x}(\mathbf{X})$

$h_{i}$

Lliq

I

K

$k_{r}$

$M$

MCS

$P$

$P p$

$P_{f}$

$R$

$T$

$u_{x}$

西
The soil cohesion strength.

The maximum liquefaction-induced lateral displacement.

The pile diameter.

The young's modulus of pile material and soil, respectively.

The flexural rigidity of the pile.

The flexural strength of the pile material and reinforced concrete, respectively.

Explicit expression of flexural and drift limit state function, respectively

Response surface.

The response surface function of moment and drift,respectively.

A chosen factor that defines the experimental/sample region.

The thickness of the liquefiable soil layer

Second moment of inertia of the pile

The number of random variables in the formulation.

The rotational stiffness of the base.

The bending moment.

Monte Carlo Simulation.

Soil pressure.

The numbers of coefficients necessary to define a polynomial.

The probability of failure.

The pile radius.

The pile thickness.

The pile head deflection. 


$\begin{array}{ll}X_{\text {all }} & \text { The allowable drift. } \\ \mathbf{x}_{C_{2}} & \text { Second center point. } \\ \mathbf{x}_{D_{1}} & \text { The coordinates of the checking point. } \\ X_{i}(i=1,2, \ldots, & \text { The } i^{\text {th }} \text { random variable } \\ k) & \\ X_{i}^{C} & \text { The coordinates of the centre point, } i . \\ \beta & \beta \text {-index =Reliability index. } \\ \varepsilon & \text { Pre-selected convergence criterion } \\ \sigma_{x_{i}} & \text { The standard deviation of a random variable } X_{i .} \\ v, v_{s} & \text { Concrete and soil Poisson's ratio, respectively. } \\ \gamma, \gamma_{s} & \text { Unit density of reinforced concrete and soil, respectively. } \\ \phi & \text { The angle of internal friction. }\end{array}$

\section{References}

1. Bradley, B., Cubrinovski, M., and Haskell, J., Probabilistic pseudo-static analysis of pile foundations in liquefiable soils. Soil Dynamics and Earthquake Engineering, 2011. 31(10): p. 1414-1425.

2. Tabesh, A. and Poulos, H., Pseudostatic approach for seismic analysis of single piles. Journal of Geotechnical and Geoenvironmental Engineering, 2001. 127(9): p. 757-765.

3. Dobry, R., Abdoun, T., O'Rourke, T., and Goh, S., Single piles in lateral spreads: Field bending moment evaluation. Journal of Geotechnical and Geoenvironmental Engineering, 2003. 129(10): p. 879-889.

4. Association, J.R., 1996, Specification for highway bridges, part v seismic design.

5. Yokoyama, K., Tamara, K., and Matsuo, O., 1997, Design methods of bridge foundation against soil liquefaction and liquefaction-induced ground flow., in 2nd Italy-Japan Workshop on Seismic Design and Retrofit of bridges: Rome, . p. 109-131. .

6. Structural, r.a.a.c. Cosmos/m

2.6; 2000: http://www.cosmosm.com

7. Haldar, A. and Mahadevan, S., Probability, reliability and statistical methods in engineering design. 2000a, New York, NY: John Wiley \& Sons.

8. Joint, C.S.S. Probabilistic model code: Part 3: Material properties. 2000; Available from: www.jcss.ethz.ch.

9. Joint, C.S.S. Probabilistic model code: Section 3.7: Soil properties. 2006; Available from: www.jcss.ethz.ch.

10.Bednar, H.H., Pressure vessel design handbook. 1986: Van Nostrand Reinhold.

11.Ellingwood, B., Development of a probability based load criterion for american national standard a58: Building code requirements for minimum design loads in buildings and other structures. 1980: U.S. Department of Commerce, National Bureau of Standards. 